\title{
Monitoring thoracic fluid content using bioelectrical impedance spectroscopy and Cole modeling
}

\author{
Silviu Dovancescu ${ }^{1,2}$, Salvatore Saporito ${ }^{1,4}$, Ingeborg H. F. Herold ${ }^{1,3}$, Hendrikus H. M. Korsten ${ }^{1,3}$, Ronald M. \\ Aarts $^{1,2}$, and Massimo Mischi ${ }^{1}$ \\ (The first two authors contributed equally to this article) \\ 1. Department of Electrical Engineering, Eindhoven University of Technology, Eindhoven, the Netherlands \\ 2. Philips Research, Eindhoven, the Netherlands \\ 3. Department of Anesthesiology and Intensive Care, Catharina Hospital Eindhoven, Eindhoven, the Netherlands \\ 4. Email any correspondence to: Salvatore.saporito@philips.com
}

\section{Abstract}

Heart failure is a chronic disease marked by frequent hospitalizations due to pulmonary fluid congestion. Monitoring the thoracic fluid status may favor the detection of fluid congestion in an early stage and enable targeted preventive measures. Bioelectrical impedance spectroscopy (BIS) has been used in combination with the Cole model for monitoring body composition including fluid status. The model parameters reflect intracellular and extracellular fluid volume as well as cell sizes, types and interactions. Transthoracic BIS may be a suitable approach to monitoring variations in thoracic fluid content.

The aim of this study was to identify BIS measures, which can be derived based on the Cole model, that are sensitive to early stages of thoracic fluid accumulation. We simulated this medical condition in healthy subjects by shifting a part of the whole blood from the periphery towards the thorax. The redistribution of blood was achieved non-invasively through leg compression using inflatable leg sleeves. We acquired BIS data before, during and after compression of the legs and examined the effect of thoracic fluid variations on parameters derived based on the Cole model and on geometrical properties of the impedance arc. Indicator dilution measurements obtained through cardiac magnetic resonance imaging were used as a reference for the changes in pulmonary fluid volume.

Eight healthy subjects were included in the study. The Cole model parameters of the study group at baseline were: $R_{0}=51.4 \pm$ $6.7 \Omega, R_{\infty}=25.0 \pm 7.0 \Omega, f_{c}=49.0 \pm 10.5 \mathrm{kHz}, \alpha=0.687 \pm 0.027$, the resistances of individual fluid compartments were $R_{E}=51.4 \pm 6.7$ $\Omega, R_{l}=50.5 \pm 22.9 \Omega$, the fluid distribution ratio was $K=1.1 \pm 0.3$, and the radius, area and depression of the arc's center were: $R=$ $15.7 \pm 1.3 \Omega, X_{C}=-8.5 \pm 1.5 \Omega, A=134.0 \pm 15.6 \Omega^{2}$. The effect of leg compression was a relatively small, reversible increase in pulmonary blood volume of $90 \pm 57 \mathrm{~mL}$. We observed significant changes in parameters associated with intracellular, extracellular and total fluid volume $\left(R_{0}:-1.5 \pm 0.9 \%, p<0.01 ; R_{\infty}:-2.1 \pm 1.1, p<\right.$ $\left.0.01 ; R_{1}:-2.6 \pm 1.6 \%, p<0.01\right)$, and in the arc's geometrical properties (R: $-1.6 \pm 1.3 \%, p<0.05 ; X_{c}:-1.7 \pm 1.5 \%, p<0.05, A$ : $-2.9 \pm 1.2 \%, p<0.01) . K$ and the parameters associated with tissue structure $f_{c}$ and $\alpha$ remained stable.

Transthoracic BIS is sensitive to small variations in intrathoracic blood volume, in particular the resistances of fluid compartments and the geometric properties of the impedance arc. Taken together with previous studies, our findings suggest that $R_{0}$ may be a suitable parameter to monitor congestion. Use of additional parameters such as $R_{l}, K, X_{c}, f_{c}$ and $\alpha$ may enable the discrimination between different types and stages of thoracic fluid accumulation and should be the focus of future research.

Keywords: Thoracic impedance, Cole model, blood shift

\section{Introduction}

Heart failure (HF) is a chronic disease that poses a major challenge to healthcare systems in developed countries. The prevalence of HF increases with age and HF has become the leading cause of hospitalization among elderly patients [1]. Most HF hospitalizations are associated with fluid congestion of the lungs, which starts before other signs and symptoms of health deprecation [2-5]. Therefore, fluid status monitoring may provide early feedback, contributing to improved management of HF patients [6]. 
The standard approach to monitor fluid status in $\mathrm{HF}$ is based on variations in body weight. However, it was found that body weight has low performance in predicting hospitalization [4] and that there is only poor correlation between symptoms and changes in body weight [7]. This may be due to the fact that the mechanism underlying fluid congestion is not limited to fluid retention, but also includes intravascular fluid redistribution towards the lungs, which is most likely to occur in the early stages of congestion [8,9]. Therefore, a more specific approach for detecting pulmonary fluid congestion that is sensitive to fluid redistribution is based on monitoring the pulmonary capillary wedge pressure (PCWP) [10]. However, the measurement of PCWP is an invasive technique, which limits its widespread use.

A different approach to monitor fluid status that may be applied non-invasively relies on measurements of bioimpedance. Living organisms are complex structures consisting of cells filled with intracellular fluid (ICF). The cells are suspended in extracellular fluids (ECF), e.g. the interstitial fluid in tissues, or blood plasma. When excited by an alternating electrical current, biologic tissues show a complex electrical bioimpedance that varies with tissue composition [11]; increased fluid volume causes the bioimpedance to decrease. Although the frequency of the applied current is not standardized for fluid status monitoring, it is commonly in the so-called " $\beta$-dispersion" range $5-1000 \mathrm{kHz}$. Due to the capacitive behavior of the cell membranes, low frequency currents pass through the ECF around the cells and high frequency currents penetrate the cell membranes passing through both ECF and ICF [12]. Hence, the sensitivity of bioimpedance measurements to different fluid compartments (ECF or ICF) depends on the excitation frequency.

The technique of measuring bioimpedance over a wide frequency range is known as bioelectrical impedance spectroscopy (BIS). The data are distributed along a circular arc (impedance locus) in the plane of complex numbers. An empirical model, known as the Cole model [13], which describes the circular arc and the frequency scale, has been widely used in order to derive electrical properties of tissue. The model parameters are associated with tissue composition, including intracellular and extracellular fluids, and tissue structure [11]. Previous studies have shown that altered tissue composition leads to changes in the impedance locus, e.g. displacement, contraction, enlargement or rotation, which are reflected by the parameters of the Cole model [14-16].

Bioimpedance measurements have been used in the assessment of body composition, including body fat, muscle mass and total body water [11, 17], as well as in monitoring hemodialysis [18-19]. These applications rely on whole body measurements obtained using a hand-to-foot electrode configuration. However, the impedance of the thorax accounts for a disproportionately small fraction of the whole body impedance (10\%) compared to body weight (4\%) [20-22]. Therefore, segmental bioimpedance measurements of the thorax have been suggested in order to maximize the sensitivity to changes in pulmonary fluid volumes [23]. Thoracic measurements have been shown to be sensitive to fluid loss after thoracentesis in patients with fluid accumulation below the lungs, in the pleural cavity $[24,25]$. Studies in HF patients with pulmonary edema, i.e. excess fluid distributed within the lungs, in the interstitium and alveoli, have shown that thoracic bioimpedance is also sensitive to fluid drained by intravenous administration of diuretics [26-28]. These studies have revealed that thoracic bioimpedance decreases once a large amount of fluid is drained from the thorax. However, these scenarios do not resemble the initial stages of pulmonary congestion, which are characterized by small volumes of excess fluid that are mainly contained in the pulmonary vasculature. The ability to detect the accumulation of these small fluid volumes may allow early medical interventions.

A major challenge of studies that use segmental bioimpedance measurements in the assessment of fluid status is the lack of an adequate reference for changes in fluid volume in the segment of interest, e.g. the lungs. This shortcoming may be addressed by magnetic resonance imaging (MRI), which is a minimally invasive technique that has the ability to quantify the pulmonary fluid volume. To this aim, MRI uses measurements of blood flow based on phase contrast MRI (PC-MRI) sequences [29], and indicator dilution measurements using gadolinium-based contrast agents in combination with dynamic contrast-enhanced MRI (DCE-MRI) sequences [30, 31]. Repeated MRI measurements may be used to correlate variations in thoracic bioimpedance with changes in intrathoracic fluid volumes.

In this study, we sought to identify bioimpedance based measures that are sensitive to small changes in thoracic fluid volumes. We induced blood displacement through pneumatic compression of the legs and measured thoracic bioimpedance using the BIS approach. We determined tissue properties based on the parameters of the Cole model and assessed their feasibility for monitoring thoracic fluid accumulation. We used MRI in order to quantify the induced changes in pulmonary blood volume.

\section{Materials and methods}

The study is part of a larger investigation aimed at comparing the effect of intrathoracic fluid variations on BIS and MRI measures. Pneumatic compression of the legs was applied as a fluid challenge to achieve a displacement of blood volume from the legs towards the thorax. Subjects underwent two repetitions of the fluid challenge paralleled by the acquisition of BIS and MRI measurements, respectively. 


\section{Study population}

The study was conducted at the Catharina Hospital Eindhoven, The Netherlands. The protocol was approved by the local medical ethics committee and was registered as a clinical trial at clinicaltrials.gov (NCT02364193). Eligible for the BIS part of the study were healthy adults (age $\geq 18$ years) without known history of cardiovascular pathologies, and with body mass index (BMI) between 18 and 25. All participants provided written informed consent at enrollment.

\section{Pneumatic leg compression}

A common physiological maneuver used to induce a gravitational displacement of blood volume from the lower extremities towards the thorax is passive leg raising (PLR) [32]. The maneuver involves the assisted raising of the legs from the horizontal. Although thoracic BIS measurements may be acquired during PLR, they may be confounded by undesired effects occurring during the maneuver: the advancement of abdominal organs towards the thorax alters the aggregated resistivity of the thoracic tissue and contributes to a deformation of the thoracic contour $[33,34]$. Similar effects occur during maneuvers that involve tilting the whole body, e.g. head down tilt [35].

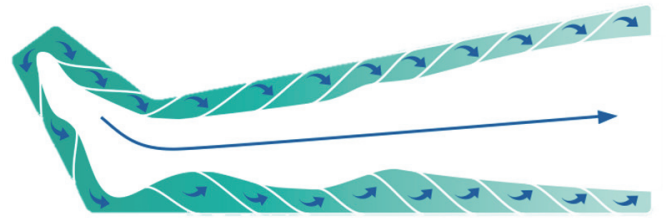

Figure 1. Schematic illustration of an inflatable leg sleeve including 12 circular air chambers. Each chamber is inflated individually to compress a section of the leg. The pressures in the individual chambers form a gradient along the leg. The highest pressure (dark green) is applied to the foot. Through external compression of the leg, a part of the peripheral blood is shifted towards the upper body.

To exclude confounding factors related to postural changes, subjects maintained a supine body posture throughout the measurement session. Variations in thoracic fluid volume were induced through external pneumatic compression of the legs by means of a Lympha-mat Digital (Bösl Medizintechnik, Aachen, Germany) gradient pump system. The system consists of an automatic air pump connected to a pair of inflatable leg sleeves each with 12 overlapping airchambers (Figure 1). Both leg sleeves are inflated in parallel: the chambers are inflated individually in temporal succession starting from the foot to the upper leg (distal to proximal). Inflation ends when the pressure in each air chamber has reached a predefined target value. In our study, the chamber pressure was distributed with a spatial gradient along the leg, such that at the end of an inflation cycle the pressure reached $100 \mathrm{mmHg}$ in the four most distal chambers, 80 $\mathrm{mmHg}$ in the four intermediate chambers, and 60 $\mathrm{mmHg}$ in the four proximal chambers. The high pressures were aimed at maximizing the blood volume displaced from the legs and the spatial gradient at preventing venous pooling in the feet. Under normal operational conditions, the compression device automatically releases the pressure simultaneously from all chambers by opening a valve once the target pressure profile has been reached, and starts a new inflation cycle. To allow sufficient time for measurements, we adapted the device to maintain the pressure profile in the leg sleeves until the manual opening of the valve.

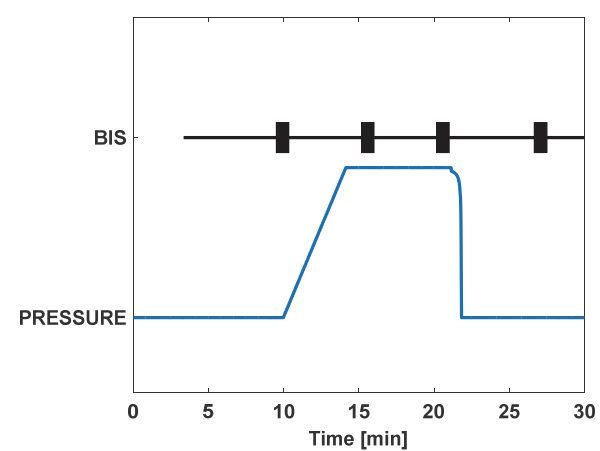

Figure 2. Leg compression and measurement protocol. The blue line is a qualitative representation of the pressure applied to the legs: the up-slope marks the gradual pressure build-up during the inflation of the leg sleeves; the downward step marks the pressure drop in the air chambers as the valves are opened for deflation. The black line represents the continuous BIS measurement, black rectangles indicate the four periods of end-expiratory breath-hold used in the analysis. (BIS = bioimpedance spectroscopy).

Before the pump was started, participants lied in supine position wearing the deflated leg sleeves for 10 minutes to achieve a physiological steady state. The progressive inflation of the leg sleeves was typically finalized in 5 minutes. The target pressure profile was kept constant for 5 minutes. The pressure was subsequently released with a manual command of the valve and participants remained in supine position without compression for 10 minutes. An overview of the compression protocol is shown in Figure 2.

\section{Bioimpedance spectroscopy}

When excited by an alternating electrical current, tissue shows a frequency dependent behavior: low frequency currents flow around the cells through the extracellular fluid, and high frequency currents are able to penetrate the cell membranes and flow through both intracellular and extracellular fluids (Figure 3a). A simplified electrical circuit that illustrate this phenomenon consists of a resistor for the extracellular path $\left(R_{E}\right)$ in parallel with a capacitor $\left(C_{M}\right)$ for the frequency 
dependent behavior of the cell membranes and a resistor for the intracellular path $\left(R_{1}\right)$ (Figure $3 b$ ).
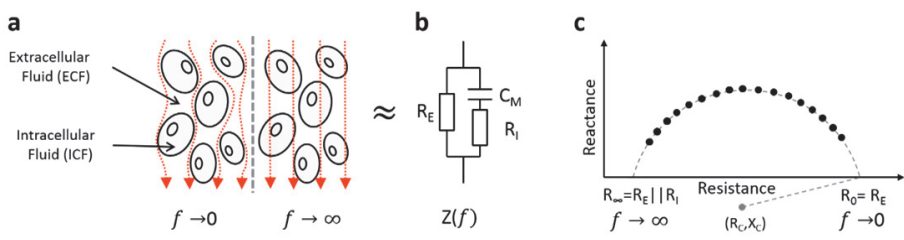

Figure 3. Principles of bioimpedance spectroscopy. (a) Flow of electrical current through biological tissue: low-frequency currents $(f \rightarrow 0)$ flow around the cells through the extracellular fluid while high-frequency currents $(f \rightarrow \infty)$ penetrate the cell membranes and flow through intracellular and extracellular fluid. (b) Simplified electrical circuit of the tissue bioimpedance consisting of two resistors and a capacitor accounting for the resistances of extracellular $\left(R_{E}\right)$ and intracellular $\left(R_{I}\right)$ fluids, and the capacitance of cell membranes $\left(C_{M}\right)$, respectively.

(c) Qualitative representation of bioimpedance spectroscopy measurement points $(Z(f))$ in the complex bioimpedance plane, the corresponding circular arc centered in $\left(R_{c}, X_{c}\right)$, and the extrapolated values at low frequencies $\left(f \rightarrow 0, Z(f)=R_{0}=R_{E}\right)$ and high frequencies $\left(f \rightarrow \infty, Z(f)=R_{\infty}=R_{E} \| R_{I}\right.$ ) according to the Cole model.

When measured over a wide frequency range, tissue bioimpedance values often describe a circular arc (impedance locus) centered below the resistance axis of the complex impedance plane (Figure 3c). The data do not accurately fit the impedance of the simplified electrical circuit (Figure $3 b$ ) because the cell membranes cannot be modelled by an ideal capacitor. To mathematically describe experimentally acquired data, Cole proposed the following empirical equation [13]

$$
Z(f)=R_{\infty}+\frac{R_{0}-R_{\infty}}{1+\left(j \frac{f}{f_{c}}\right)^{\alpha}}
$$

where $Z(f)$ is the complex bioimpedance as a function of the frequency $f, R_{0}$ is the impedance at very low frequencies $(f \rightarrow 0), R_{\infty}$ is the impedance at very high frequencies $(f \rightarrow \infty), f_{c}$ is the frequency corresponding to the peak of the circular arc where the reactance reaches its maximum, and $\alpha(0<\alpha \leq 1)$ is a dimensionless exponent which defines the depression of the circular arc with respect to the resistance axis. Spectroscopic bioimpedance measurements can, hence, be described using the four Cole model parameters $R_{0}$, $R_{\infty}, f_{c}$ and $\alpha$.

Based on the Cole model, several measures may be used to characterize and monitor changes in tissue composition. The fluid content of different fluid compartments is reflected by the resistance of the extracellular fluids $\left(R_{E}=R_{0}\right)$, the aggregated resistance of intracellular and extracellular fluids $\left(R_{\infty}=\right.$ $\left.R_{E} \| R_{I}\right)$, the resistance of intracellular fluids $\left(R_{I}=\right.$
$\left.R_{E} R_{\infty} /\left(R_{E}-R_{\infty}\right)\right)$, or the ratio between the resistance of extracellular and intracellular fluids as an approximate value of the fluid distribution ( $\left.K=R_{E} / R_{I}=R_{0} / R_{\infty}-1\right)$ ) [36]. The tissue structure, including cell types, sizes and interactions, is reflected by the characteristic frequency of the tissue $\left(f_{c}\right)$ and the distribution of relaxation times $(\alpha)$. More details about the model and the interpretation of its parameters can be found in [37].

\section{Data acquisition}

TI was monitored using a commercially available BIS device SFB7 (ImpediMed Ltd, Brisbane, Australia). The device measures bioimpedance at 256 frequencies in the range $3-1000 \mathrm{kHz}$ using a tetra polar electrode configuration (two electrodes for the application of an excitation current, and two for voltage measurements). A transthoracic electrode placement was used to maximize the sensitivity of the measurement to pulmonary fluid [23]: an excitation and a measurement electrode were placed on either side of the chest, along the axillary lines at the level of the $5^{\text {th }}$ intercostal spaces (Figure 4). The distance between neighboring electrodes was approximately $5 \mathrm{~cm}$. BIS measurements were acquired throughout the leg compression protocol in continuous mode for 30 minutes at a rate of 40 bioimpedance spectra/minute. Subjects were asked to perform end-expiratory breath hold at specific time instances: at baseline (BL), after maximum inflation at steady pressure (SP), before deflation (BD), after deflation (AD). The breath hold intervals enable an assessment of a steady state $\mathrm{TI}$ without respiratory variations.

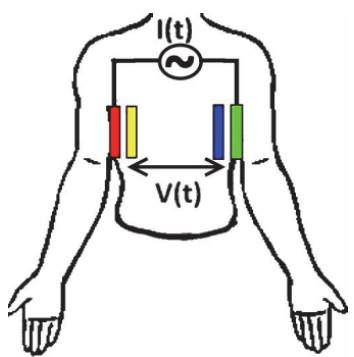

Figure 4. Electrode placement for trans-thoracic bioimpedance measurements: two electrodes (red and green) are used for the application of an excitation current $(I(t))$, and two electrodes for the measurement of the voltage drop $(\mathrm{V}(\mathrm{t}))$.

\section{Data analysis}

Each bioimpedance spectrum was used to derive Cole model parameters $\left(R_{0}, R_{\infty}, f_{c}, \alpha\right)$, resistances of the extracellular and intracellular fluid compartments $\left(R_{E}\right.$, $\left.R_{I}\right)$, the ratio $K$, and the geometrical properties of the circular arc: radius $(R)$, depression of the center with respect to the resistance axis $\left(X_{C}\right)$, and arc segment area $(A)$. To this aim, the Cole model was fitted to the BIS data using the software Biolmp 5.4 (ImpediMed Ltd, Brisbane, Australia) which implements a multiple linear 
regression algorithm. Values corresponding to the intervals of end-expiratory breath hold were averaged to minimize measurement errors and the variability due to the cardiac cycle. To assess the effect of thoracic fluid changes on model parameters, we compared the breath-hold intervals around the inflation (BL-SP) and deflation (BD-AD) of the leg sleeves using the paired Student's t-test. Normal distribution of the samples was verified using the Lillieforce test. A p-value $<0.05$ was considered statistically significant. The data analysis was performed in Matlab ${ }^{\circledR}$ 2015b (Natick, MA, USA).

\section{Cardiac magnetic resonance imaging}

To confirm and quantify the changes in intra-thoracic blood volume induced by the fluid challenge we used MRI-based indicator dilution measurements. Each subject participated in a repetition of the pneumatic leg compression protocol paralleled by minimally-invasive MRI measurements. MRI was performed on a $1.5 \mathrm{~T}$ Ingenia MR scanner (Philips Healthcare, Best, The Netherlands) with a phased-array cardiac coil.

To enable the estimation of pulmonary blood volume, paired measurements of PC-MRI and DCE-MRI were acquired repeatedly before, during and after pneumatic leg compression. The PC-MRI measurements were used to determine stroke volume (SV). Each DCEMRI measurement included intravenous injections of $0.2 \mathrm{mmol}$ Dotarem (Guerbet, Villepinte, France) contrast agent diluted into $5 \mathrm{~mL}$ saline solution. The injections were administered intravenously at the rate of $5 \mathrm{~mL} / \mathrm{s}$ using an automated MR injector (Medrad, Indianapolis, US). Following the injection, a dynamic series of images was acquired. Based on the linear relationship between CA concentration and MR signal [30], indicator dilution curves were derived and used to fit the local density random walk (LDRW) model [38]. Intra-thoracic transit time (ITT) was determined as the difference between the mean transit times of the left and the right ventricular indicator dilution curves. Heart rate $(H R)$ was derived from the ECG signal used for gating. Finally, intra-thoracic blood volume was obtained using the formula: ITBV $=S V \cdot H R \cdot I T T$. The detailed processing methods for deriving the MRI-based measures can be found in [31].

\section{Results}

A total of 8 healthy volunteers aged $39 \pm 13$

participated in the study. All subjects were males with a body mass index (BMI) of $23.9 \pm 2.3 \mathrm{~kg} / \mathrm{m}^{2}$ and a body surface area (BSA) of $1.95 \pm 0.12 \mathrm{~m}^{2}$.

The Cole model parameters of the study group at baseline were $R_{0}=51.4 \pm 6.7 \Omega, R_{\infty}=25.0 \pm 7.0 \Omega, f_{c}=$ $49.0 \pm 10.5 \mathrm{kHz}, \alpha=0.687 \pm 0.027$, the resistances of individual fluid compartments were $R_{E}=51.4 \pm 6.7 \Omega$,
$R_{I}=50.5 \pm 22.9 \Omega$, the fluid distribution $K=1.12 \pm 0.3$, and the geometrical characteristics of the impedance locus were $R=15.7 \pm 1.3 \Omega, X_{C}=-8.5 \pm 1.5 \Omega, A=134.0$ $\pm 15.6 \Omega^{2}$

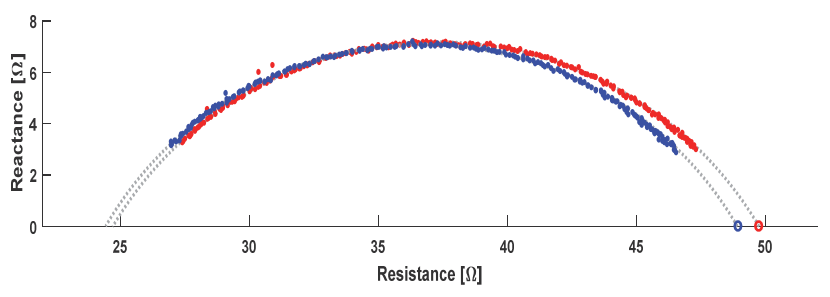

Figure 5. Bioimpedance spectra before (red) and after (blue) leg compression. Dashed circular arcs mark the extrapolated values according to the Cole model.

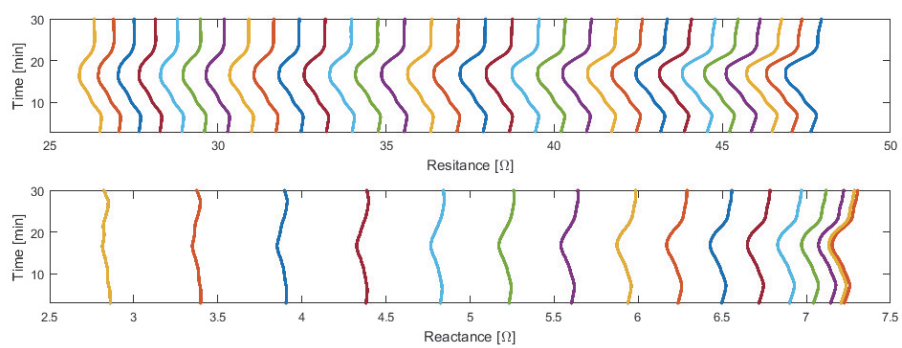

Figure 6. Bioimpedance trends at multiple frequencies in one study subject. Each line is a local regression curve indicating the temporal evolution of resistance (top) and reactance (bottom) for a single frequency on the Cole arc. Local regression is used to suppress cardio-respiratory artifacts. The trends show a linear decrease in bioimpedance (resistance and reactance) during the inflation of the leg sleeves, a plateau during the maintenance of the target pressure, and a return to baseline after the release of pressure from the leg sleeves. The decrease in resistance and reactance reflects the translation and the contraction of the Cole arc, respectively.

Pneumatic leg compression led to a reversible increase in pulmonary blood volume in the order of 90 $\pm 57 \mathrm{~mL}$ according to our MRI measurements.

Compression of the legs also had a reversible effect on transthoracic bioimpedance, which decreased (Figure 6). Examples of bioimpedance spectra before and after the inflation of the leg sleeves are shown in Figure 5. During inflation, the Cole arc contracted and moved progressively to the upper-left of the complex bioimpedance plane. In particular, the resistances $R_{0}$, $R_{\infty}$ and $R_{I}$, and the geometrical properties of the $\operatorname{arc} R$, $X_{C}$, and $A$ decreased until the target pressure profile was reached $\left(R_{0}:-1.5 \pm 0.9 \%, \mathrm{p}<0.01 ; R_{\infty}:-2.1 \pm 1.1\right.$ $\%, \mathrm{p}<0.01 ; R_{I}:-2.6 \pm 1.6 \%, \mathrm{p}<0.01 ; R:-1.6 \pm 1.3 \%, \mathrm{p}$ $<0.05 ; X_{C}:-1.7 \pm 1.5 \%, \mathrm{p}<0.05, A:-2.9 \pm 1.2 \%, \mathrm{p}<$ $0.01)$. The opposite effect occurred rapidly upon pressure release $\left(R_{0}:+1.4 \pm 0.8 \%, \mathrm{p}<0.01 ; R_{\infty}:+1.6 \pm\right.$ $0.9, \mathrm{p}<0.01 ; R_{I}:+1.8 \pm 1.3 \%, \mathrm{p}<0.01 ; R:+1.3 \pm 0.9 \%$, $\mathrm{p}<0.01 ; X_{C}:+1.7 \pm 1.0 \%, \mathrm{p}<0.01, A:+2.0 \pm 1.9 \%, \mathrm{p}<$ $0.05)$. The ratio $K$ did not change significantly during the two transitions, suggesting that the fluid distribution 
between the intracellular and extracellular compartments remained stable.

There was no significant change in $f_{c}$ to indicate a rotation of the Cole arc with the induced variations in thoracic blood volume. There was also no significant change in $\alpha$ that would indicate changes in the distribution of time constants in the thoracic tissue as a result of the induced fluid displacements. Figure 7 shows the typical temporal evolution of the analyzed Cole model parameters and the geometrical properties of the arc throughout the compression protocol for one subject.
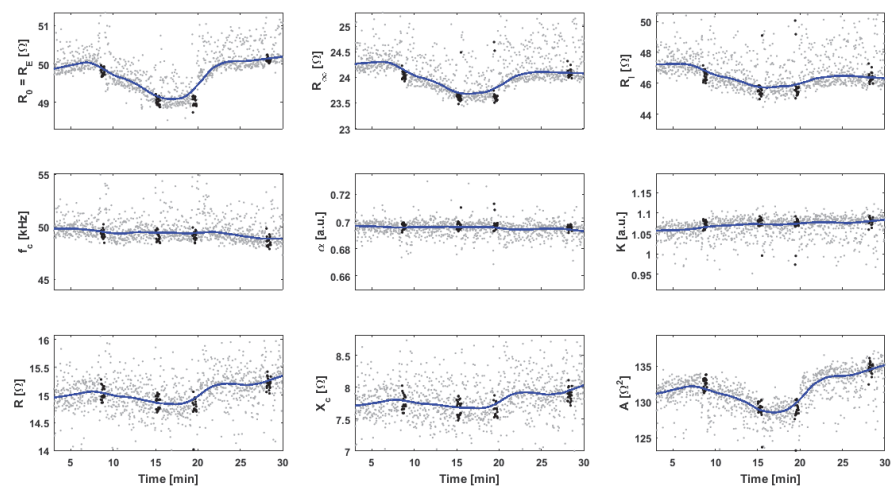

Figure 7. Evolution of BIS measures throughout the leg compression protocol in one subject. Gray dots are values obtained during periods of free breathing, black dots are values corresponding to periods of end-expiratory breath hold before, during and after leg compression, blue lines are local regression curves fitted to the data. ( $R_{0}$ : resistance at low frequencies; $R_{\infty}$ : resistance at high frequencies; $R_{\mathrm{l}}$ : resistance intracellular fluids; $f_{c}$ : characteristic frequency of the tissue; $\alpha$ : distribution of relaxation times; $\mathrm{K}$ : ratio between resistance of extra- and intra-cellular fluids; $R$ : radius of impedance $\operatorname{arc} ; X_{c}$ : depression of the center of impedance arc; A: impedance arc segment area. BIS = bioimpedance spectroscopy).

\section{Discussion}

The aim of this study was to evaluate the sensitivity of thoracic bioimpedance measured using the BIS technique to variations in intrathoracic fluid content induced through redistribution of peripheral blood. By increasing the intrathoracic blood volume, we simulated, in a controlled environment, effects of incipient pulmonary congestion that is characterized by vascular fluid redistribution $[8,9]$. By decreasing the intrathoracic blood volume, we simulated decongestion that, in HF, is typically achieved through administration of diuretics. Redistribution of blood was achieved through external compression of the legs in order to minimize the potential confounding effects associated with changes in body posture and organ shift on BIS measures $[33,34]$. To quantify the changes in thoracic fluid volume induced by our fluid challenge we used MRI, which confirmed that the changes in the pulmonary circulation were small, in the order of $90 \mathrm{~mL}$, and reversible. By design, our compression protocol led to a redistribution of whole blood from the legs without altering blood composition.
Due to the compliance of the pulmonary vessels, it is likely that the small increase in fluid volume did not lead to extravascular leakage while the external pressure was maintained on the legs.

When a fraction of the peripheral blood was shifted to the thorax, we observed that the circular arc contracted and moved towards lower impedance values. This suggests that the increase in intravascular fluid led to an increase in the aggregated conductivity of the thoracic tissues. The opposite effect was observed when compression ended, i.e. the circular arc returned towards the original position and size. Of the four model parameters $\left(R_{0}, R_{\infty}, f_{c}, \alpha\right)$, only the extrapolated resistances at 0 and infinite frequency $\left(R_{0}\right.$ and $R_{\infty}$ ) showed significant changes and were, thus, sensitive to the small variations in pulmonary blood volume. When considering the equivalent electrical circuit, we observed significant changes in intracellular and extracellular resistance $\left(R_{E}\right.$ and $\left.R_{I}\right)$, which both followed similar trends, inverse to the thoracic fluid variation. This suggests that $R_{E}$ and $R_{I}$ reflect the respective variations in blood plasma (extracellular fluid compartment) and complete blood count (intracellular fluid compartment). Our observation that the ratio $K$, as a measure of fluid distribution, remained stable during the fluid challenge is in agreement with the fact that blood plasma and intracellular fluid each account for approxi-mately half of the total blood volume and that there is no significant difference in resistivity between intracellular and extracellular fluids [36].

Given that the blood composition was not altered during our experiment, it is unlikely that the temporary displacement of whole blood towards the thorax may have caused osmotic in-equilibrium, which leads to fluid shift between the intracellular and the extracellular fluid compartments. According to the Cole theory, fluid shift through the cellular membranes would result in opposing trends in $R_{E}$ and $R_{I}$, which were not observed in our study. However, the absence of fluid shift could not be verified based on our MRI measures, as these do not enable a discrimination between intracellular and extracellular fluid volumes.

The characteristic frequency of most muscle tissue is $\mathbf{5 0}$ $\mathrm{kHz}$, which is approximately equal to the baseline value observed in our study group. The characteristic frequency of whole blood is $2 \mathrm{MHz}[37,39]$, which exceeds the frequency range used for our BIS measurements. Taken separately, blood and tissue can be considered as independent Cole systems with $f_{c}$ values that differ by orders of magnitude. An aggregated system obtained by increasing the blood volume in tissue as was done in our study can, theoretically, reveal two circular arcs depending on the relative volume of blood compared to tissue and the frequency range used for the BIS measurement [37]. A second circular arc could not be distinguished in our study data and the standard error of estimate for the model fit was stable. Furthermore, $f_{c}$ remained stable throughout the fluid challenge, i.e. the 
impedance locus did not rotate. This suggests that the frequency scale of thoracic tissue is a dominant mechanism and that the impedance arc and the frequency scale were not affected by the relatively small increase in thoracic blood volume in the frequency range used for our measurements.

The parameter $\alpha$, which is used to model the depression of the Cole arc with respect to the resistance axis did not vary significantly during our experiment. Geometrically, $\alpha$ depends on the depression of the arc's center with respect to the resistance axis and on the radius $(R)$, which were both affected by our experiment. The decrease in bioimpedance expressed by a contraction of the Cole arc (reduction in $R$ ) was compensated by an upward shift of the arc's center. $\alpha$ is regarded as a measure of distribution of relaxation times due the differences in cell sizes, types and interactions in the various structures making up the heterogeneous tissue [37]. Hence, our results indicate that relatively small variations in thoracic blood volume have no significant effect on the distribution of relaxation times of the thoracic tissue. However, the vertical displacement of the arc's center in the absence of $\alpha$ variations suggests that the parameter $X_{C}$ may be sensitive to specific changes in tissue structure, such as variations in blood content, and should therefore be the object of further investigation.

Previous studies have used Cole modeling for BIS measurements obtained with a transthoracic electrode configuration in the context of thoracic fluid accumulation $[24,26]$. Only a limited number of parameters were available for comparison with our results $\left(R_{E}\right.$ and $\left.R_{I}\right)$. In contrast to our in-vivo simulation of early stages of pulmonary congestion, these studies have investigated late stages of thoracic fluid accumulation in patients treated for $\mathrm{HF}$ decompensation or pleural effusion. In HF, the main characteristics of advanced fluid congestion are a positive fluid balance and elevated pulmonary pressures, which lead to a leakage of vascular extracellular fluid (blood plasma) into the extravascular space (interstitium) and subsequently into the alveoli resulting in pulmonary edema. During this process, fluid may also shift between the extracellular and intracellular space, through cellular membranes, due to changes in trans-membrane ion concentration. HF patients were studied while undergoing inpatient treatment with intravenous diuretics after hospital admission with pulmonary edema [26]. Because direct, non-invasive measures of thoracic fluid or excess fluid volume are typically not available in standard clinical practice, changes in body weight were used as a surrogate. The study reported that an average weight loss of $1.6 \mathrm{~kg}$ during diuretic therapy was reflected into a transthoracic impedance change of $R_{E}:+25 \%$ and $R_{I}:+10 \%$ relative to the discharge ("dry") value. A study in patients with pleural effusion reported changes of $R_{E}:+10 \%$ and $R_{I}:+2 \%$ relative to the "dry" value for an average of $1.2 \mathrm{~L}$ of fluid removed from the thorax [24]. Pleural effusion consists mainly of extracellular fluid and is located below the lungs. The results of these studies are in agreement with our finding that $R_{E}$ and $R_{I}$ are sensitive to changes in thoracic fluid volume and are expected to decrease as the fluid volume increases.

Based on the results reported by the two studies in $\mathrm{HF}$ and pleural drainage patients $[26,24]$, we derived the ratio $K=R_{E} / R_{I}$, which may be used as a measure of fluid distribution because the resistivity of intracellular and extracellular fluids is stable [39]. The expected effect of an extracellular fluid shift into the intracellular space while the total fluid volume remains constant, is an increase in $R_{E}$, a decrease in $R_{I}$, and an increase in $K$. Furthermore, $K$ may also increase as a result of increased $R_{E}$ due to drainage of extracellular fluid from tissue. In HF patients, $\mathrm{K}$ increased from 0.57 to 0.63 and in pleural effusion patients from 0.57 to $0.62[24,26]$. The pleural effusion study included a separate control group of healthy subjects with $K=0.94$, which is comparable to our results. $K$ was lower in patients suffering from thoracic fluid accumulation most likely due to the excess of extracellular fluid, which is reflected into lower $R_{E}$ values. The fact that, after therapy, $K$ of patients remained low compared to the "healthy" value indicates that the patients were discharged before the "dry" state was reached. Taken together, the results of these studies suggest that $R_{E}$ is sensitive to fluid accumulation both in the vascular and the extravascular compartments. Furthermore, monitoring $R_{E}, R_{I}$ and $K$ may aid discharge planning and help to discriminate between excess fluids which consist mainly of extracellular fluid (e.g. interstitial, alveolar or pleural fluids) or both intra- and extracellular fluid (e.g. whole blood).

The Cole model has also been studied in combination with whole body BIS measurements for monitoring total body water during sessions of hemodialysis $[15,18,19]$. The studies report a general increase in bioimpedance, i.e. a displacement of the Cole arc towards higher values following fluid drainage. In particular, a study that reported multiple variables derived based on the Cole model found changes by $R_{E}:+26 \%, R_{I}: 0 \%, f_{C}:+8 \%$ and $\alpha:+175 \%$ for an average weight change of $-4 \mathrm{~kg}$ due to ultrafiltration [15]. In contrast to our results, $R_{I}$ did not change significantly before and after the dialysis session probably because fluid removed through ultrafiltration is mainly from the extracellular space. The authors acknowledge that the changes in $f_{c}$ and $\alpha$ require further investigation [15].

\section{Conclusions}

In this study, we simulated fluid redistribution as an early mechanism on pulmonary congestion in order to investigate the effects on measures derived from transthoracic BIS measurements according to the Cole model. Using external leg compression, we achieved a reversible increase in 
pulmonary blood volume in the order of $90 \mathrm{~mL}$ (measured by MRI). Geometric characteristics of the impedance locus $\left(R, X_{C}, A\right)$ and the Cole variables reflecting fluid content in the extracellular and intracellular compartments $\left(R_{0}=\right.$ $R_{E}, R_{I}, R_{\infty}$ ) were sensitive to the small variations in thoracic blood volume. The ratio $K$ denoting fluid distribution, and variables reflecting tissue structure; $f_{c}, \alpha$, remained stable. Our results, together with previous studies, suggest that $R_{0}$ may be an adequate parameter for monitoring early to late stages thoracic fluid accumulation due to its sensitivity to vascular, interstitial, alveolar and pleural fluids. Our early findings on the correlation between $R_{E}$ and pulmonary blood volume assessed by MRI are available in [40]. Use of additional parameters e.g. $R_{I}, K, f_{c}, \alpha, X_{C}$ may enable the discrimination between different types and stages of thoracic fluid and should be the focus of future research.

\section{References}

1. F. Zannad, N. Agrinier, and F. Alla, "Heart failure burden and therapy," Eur. Eur. Pacing Arrhythm. Card. Electrophysiol. J. Work. Groups Card. Pacing Arrhythm. Card. Cell. Electrophysiol. Eur. Soc. Cardiol., vol. 11 Suppl 5, pp. v1-9, Nov. 2009. https://doi.org/10.1093/europace/eup304

2. P. B. Adamson, A. Magalski, F. Braunschweig, M. Böhm, D. Reynolds, D. Steinhaus, A. Luby, C. Linde, L. Ryden, B. Cremers, T. Takle, and T. Bennett, "Ongoing right ventricular hemodynamics in heart failure: clinical value of measurements derived from an implantable monitoring system," J. Am. Coll. Cardiol., vol. 41, no. 4, pp. 565-571, Feb. 2003. https://doi.org/10.1016/\$0735-1097(02)02896-6

3. C.-M. Yu, L. Wang, E. Chau, R. H.-W. Chan, S.-L. Kong, M.-O. Tang, J. Christensen, R. W. Stadler, and C.-P. Lau, "Intrathoracic Impedance Monitoring in Patients With Heart Failure," Circulation, vol. 112, no. 6, pp. 841-848, 2005. https://doi.org/10.1161/CIRCULATIONAHA.104.492207

4. S. I. Chaudhry, Y. Wang, J. Concato, T. M. Gill, and H. M. Krumholz, "Patterns of Weight Change Preceding Hospitalization for Heart Failure," Circulation, vol. 116, no. 14, pp. 1549-1554, Oct. 2007. https://doi.org/10.1161/CIRCULATIONAHA.107.690768

5. M. Gheorghiade, F. Follath, P. Ponikowski, J. H. Barsuk, J. E. A. Blair, J. G. Cleland, K. Dickstein, M. H. Drazner, G. C. Fonarow, T. Jaarsma, G. Jondeau, J. L. Sendon, A. Mebazaa, M. Metra, M. Nieminen, P. S. Pang, P. Seferovic, L. W. Stevenson, D. J. van Veldhuisen, F. Zannad, S. D. Anker, A. Rhodes, J. J. V. McMurray, and G. Filippatos, "Assessing and grading congestion in acute heart failure: a scientific statement from the Acute Heart Failure Committee of the Heart Failure Association of the European Society of Cardiology and endorsed by the European Society of Intensive Care Medicine," Eur. J. Heart Fail., vol. 12, no. 5, pp. 423-433, 2010. https://doi.org/10.1093/eurjhf/hfq045

6. C. M. O'Connor, W. G. Stough, D. S. Gallup, V. Hasselblad, and M. Gheorghiade, "Demographics, Clinical Characteristics, and Outcomes of Patients Hospitalized for Decompensated Heart Failure: Observations From the IMPACT-HF Registry," J. Card.
Fail., vol. 11, no. 3, pp. 200-205, Apr. 2005.

https://doi.org/10.1016/j.cardfail.2004.08.160

7. J. E. A. Blair, S. Khan, M. A. Konstam, K. Swedberg, F. Zannad, J. C. Burnett, L. Grinfeld, A. P. Maggioni, J. E. Udelson, C. A. Zimmer, J. Ouyang, C.-F. Chen, and M. Gheorghiade, "Weight changes after hospitalization for worsening heart failure and subsequent re-hospitalization and mortality in the EVEREST trial," Eur. Heart J., vol. 30, no. 13, pp. 1666-1673, Jul. 2009. https://doi.org/10.1093/eurheartj/ehp144

8. G. Cotter, G. M. Felker, K. F. Adams, O. Milo-Cotter, and C. M. O'Connor, "The pathophysiology of acute heart failure-Is it all about fluid accumulation?," Am. Heart J., vol. 155, no. 1, pp. 9-18, Jan. 2008. https://doi.org/10.1016/j.ahj.2006.02.038

9. G. Cotter, M. Metra, O. Milo-Cotter, H. C. Dittrich, and M. Gheorghiade, "Fluid overload in acute heart failure - Redistribution and other mechanisms beyond fluid accumulation," Eur. J. Heart Fail., vol. 10, no. 2, pp. 165-169, 2008. https://doi.org/10.1016/j.ejheart.2008.01.007

10. W. T. Abraham, P. B. Adamson, R. C. Bourge, M. F. Aaron, M. R. Costanzo, L. W. Stevenson, W. Strickland, S. Neelagaru, N. Raval, S. Krueger, S. Weiner, D. Shavelle, B. Jeffries, and J. S. Yadav, "Wireless pulmonary artery haemodynamic monitoring in chronic heart failure: a randomised controlled trial," The Lancet, vol. 377, no. 9766, pp. 658-666, Feb. 2011. https://doi.org/10.1016/S0140-6736(11)60101-3

11. T. K. Bera, "Bioelectrical Impedance Methods for Noninvasive Health Monitoring: A Review", J. Med. Eng., vol. 2014, p. e381251, Jun. 2014. https://doi.org/10.1155/2014/381251

12. B. H. Cornish, B. J. Thomas, and L. C. Ward, "Improved prediction of extracellular and total body water using impedance loci generated by multiple frequency bioelectrical impedance analysis," Phys. Med. Biol., vol. 38, no. 3, p. 337, 1993. https://doi.org/10.1088/0031-9155/38/3/001

13. K. S. Cole, "Permeability and impermeability of cell membranes for ions," Cold Spring Harb. Symp. Quant. Biol., vol. 8, pp. 110-122, 1940. https://doi.org/10.1101/SQB.1940.008.01.013

14. A. Piccoli, G. Pastori, M. Guizzo, M. Rebeschini, A. Naso, and C. Cascone, "Equivalence of information from single versus multiple frequency bioimpedance vector analysis in hemodialysis," Kidney Int., vol. 67, no. 1, pp. 301-313, 2005. https://doi.org/10.1111/j.1523-1755.2005.00083.x

15. O. I. Al-Surkhi, P. J. Riu, F. F. Vazquez, and J. Ibeas, "Monitoring Cole-Cole parameters during haemodialysis (HD)," in 2007 29th Annual International Conference of the IEEE Engineering in Medicine and Biology Society, 2007, pp. 2238-2241. https://doi.org/10.1109/IEMBS.2007.4352770

16. M. Y. Jaffrin, M. Maasrani, A. Le Gourrier, and B. Boudailliez, "Extra-and intracellular volume monitoring by impedance during haemodialysis using Cole-Cole extrapolation," Med. Biol. Eng. Comput., vol. 35, no. 3, pp. 266-270, 1997. https://doi.org/10.1007/BF02530048

17. P. Deurenberg, M. Deurenberg-Yap, and F. J. M. Schouten, "Validity of total and segmental impedance measurements for prediction of body composition across ethnic population groups," Eur. J. Clin. Nutr., vol. 56, no. 3, pp. 214-220, Mar. 2002. https://doi.org/10.1038/sj.ejcn.1601303 
18. O. I. Al-Surkhi, P. J. Riu, and M. Y. Jaffrin, "Monitoring body fluid shifts during haemodialysis (HD) using electrical bioimpedance measurments," in 2011 1st Middle East Conference on Biomedical Engineering, 2011, pp. 108-113. https://doi.org/10.1109/MECBME.2011.5752077

19. A. Piccoli, "Estimation of fluid volumes in hemodialysis patients: comparing bioimpedance with isotopic and dilution methods," Kidney Int., vol. 85, no. 4, pp. 738-741, Apr. 2014. https://doi.org/10.1038/ki.2013.434

20. Lukaski H C and Scheltinga R M, "Improved sensitivity of the tetrapolar bioelectrical impedance method to assess fluid status and body composition: Use of proximal electrode placement," Age Nutr, vol. 5, pp. 123-9.

21. L. Z. Coppini, D. L. Waitzberg, and A. C. L. Campos, "Limitations and validation of bioelectrical impedance analysis in morbidly obese patients," Curr. Opin. Clin. Nutr. Metab. Care, vol. 8, no. 3, pp. 329-332, May 2005. https://doi.org/10.1097/01.mco.0000165013.54696.64

22. M. Dehghan and A. T. Merchant, "Is bioelectrical impedance accurate for use in large epidemiological studies?," Nutr. J., vol. 7, p. 26, 2008. https://doi.org/10.1186/1475-2891-7-26

23. L. Beckmann, D. van Riesen, and S. Leonhardt, "Optimal electrode placement and frequency range selection for the detection of lung water using bioimpedance spectroscopy," Conf. Proc. Annu. Int. Conf. IEEE Eng. Med. Biol. Soc. IEEE Eng. Med. Biol. Soc. Conf., vol. 2007, pp. 2685-2688, 2007. https://doi.org/10.1109/IEMBS.2007.4352882

24. M. D. Zink, S. Weyer, ren, K. Pauly, A. Napp, M. Dreher, S. Leonhardt, N. Marx, P. Schauerte, and K. Mischke, "Feasibility of Bioelectrical Impedance Spectroscopy Measurement before and after Thoracentesis," BioMed Res. Int., vol. 2015, p. e810797, Mar. 2015. https://doi.org/10.1155/2015/810797

25. M. Ulbrich, J. Muhlsteff, M. Zink, S. Leonhardt, and M. Walter, "Feasibility of impedance cardiography to assess hemodynamic changes and fluid loss related to pleural drainage," in Computing in Cardiology Conference (CinC), 2013, 2013, pp. 663-666.

26. S. Weyer, M. D. Zink, T. Wartzek, L. Leicht, K. Mischke, T. Vollmer, and S. Leonhardt, "Bioelectrical impedance spectroscopy as a fluid management system in heart failure," Physiol. Meas., vol. 35, no. 6, pp. 917-930, Jun. 2014. https://doi.org/10.1088/0967-3334/35/6/917

27. D. Freimark, M. Arad, R. Sokolover, S. Zlochiver, and S. Abboud, "Monitoring lung fluid content in CHF patients under intravenous diuretics treatment using bio-impedance measurements," Physiol. Meas., vol. 28, no. 7, pp. S269-S277, Jul. 2007. https://doi.org/10.1088/0967-3334/28/7/S20

28. I. Cuba-Gyllensten, P. Gastelurrutia, J. Riistama, R. Aarts, J. Nuez, J. Lupon, and A. Bayes-Genis, "A novel wearable vest for tracking pulmonary congestion in acutely decompensated heart failure," Int. J. Cardiol., vol. 177, no. 1, pp. 199-201, Nov. 2014. https://doi.org/10.1016/j.ijcard.2014.09.041

29. D. T. H. Wong, K.-J. Lee, S.-J. Yoo, G. Tomlinson, and L. GrosseWortmann, "Changes in systemic and pulmonary blood flow distribution in normal adult volunteers in response to posture and exercise: a phase contrast magnetic resonance imaging study," J. Physiol. Sci. JPS, vol. 64, no. 2, pp. 105-112, Mar. 2014. https://doi.org/10.1007/s12576-013-0298-z

30. M. Mischi, H. C. M. van den Bosch, J. A. den Boer, J. Verwoerd, R. J. E. Grouls, C. H. Peels, and H. H. M. Korsten, "Intra-thoracic blood volume measurement by contrast magnetic resonance imaging," Magn. Reson. Med., vol. 61, no. 2, pp. 344-353, Feb. 2009. https://doi.org/10.1002/mrm.21824

31. S. Saporito, I. H. F. Herold, P. Houthuizen, H. C. M. van Den Bosch, J. A. Den Boer, H. H. M. Korsten, H. C. van Assen, and M. Mischi, "Model-Based Characterization of the Transpulmonary Circulation by Dynamic Contrast-Enhanced Magnetic Resonance Imaging in Heart Failure and Healthy Volunteers," Invest. Radiol., vol. 51, no. 11, pp. 720-727, Nov. 2016. https://doi.org/10.1097/RLI.0000000000000304

32. X. Monnet, M. Rienzo, D. Osman, N. Anguel, C. Richard, M. R. Pinsky, and J.-L. Teboul, "Passive leg raising predicts fluid responsiveness in the critically ill," Crit. Care Med., vol. 34, no. 5, pp. 1402-1407, May 2006. https://doi.org/10.1097/01.CCM.0000215453.11735.06

33. V. F. Cassola, V. J. de M. Lima, R. Kramer, and H. J. Khoury, "FASH and MASH: female and male adult human phantoms based on polygon mesh surfaces: I. Development of the anatomy," Phys. Med. Biol., vol. 55, no. 1, p. 133, Jan. 2010. https://doi.org/10.1088/0031-9155/55/1/009

34. S. Dovancescu, A. Torabi, T. Mabote, J. Caffarel, E. Kelkboom, R. Aarts, E. Korsten, and J. Cleland, "Sensitivity of a Wearable Bioimpedance Monitor to Changes in the Thoracic Fluid Content of Heart Failure Patients," Comput. Cardiol., 2013.

35. A. C. Henderson, G. K. Prisk, D. L. Levin, S. R. Hopkins, and R. B. Buxton, "Characterizing pulmonary blood flow distribution measured using arterial spin labeling," NMR Biomed., vol. 22, no. 10, pp. 1025-1035, Dec. 2009. https://doi.org/10.1002/nbm.1407

36. H. Kanai, M. Haeno, and K. Sakamoto, "Electrical measurement of fluid distribution in legs and arms," in Medical Progress through technology, Springer, 1987, pp. 159-170.

37. S. Grimnes, Bioimpedance and bioelectricity basics. $3^{\text {rd }}$ ed. Boston, MA: Elsevier, 2015.

38. M. Mischi, T. A. Kalker, and E. H. Korsten, "Contrast echocardiography for pulmonary blood volume quantification," IEEE Trans. Ultrason. Ferroelectr. Freq. Control, vol. 51, no. 9, pp. 1137-1147, Sep. 2004. https://doi.org/10.1109/TUFFC.2004.1334846

39. H. Kanai, K. Sakamoto, and M. Haeno, "Electrical measurement of fluid distribution in human legs: estimation of extra- and intra-cellular fluid volume," J. Microw. Power, vol. 18, no. 3, pp. 233-243, Sep. 1983. https://doi.org/10.1080/16070658.1983.11689328

40. S. Saporito, S. Dovancescu, I. H. F. Herold, H. C. M. van Den Bosch, H. C. van Assen, R. M. Aarts, H. H. M. Korsten, and M. Mischi, "Comparison of cardiac magnetic resonance imaging and bio-impedance spectroscopy for the assessment of fluid displacement induced by external leg compression," Physiological measurement vol 38 no.1, pp. 15-32, 2017. https://doi.org/10.1088/1361-6579/38/1/15 